\title{
Xiao Zheng San Jie Granules in the Treatment of Qi Deficiency and Blood Stasis Syndrome in Patients With Diabetic Kidney Disease Stage IV: Protocol for a Randomised, Double-blind, Placebo-controlled, Multi-centre Trial
}

\section{Sinan Ai}

Beijing University of Chinese Medicine Affiliated Dongzhimen Hospital

\section{Yizhen Han}

Beijing University of Chinese Medicine Affiliated Dongzhimen Hospital

\section{Yabin Gao}

Hainan Province Hospital of Traditional Chinese Medicine

\section{Yaoxian Wang}

Beijing University of Chinese Medicine Affiliated Dongzhimen Hospital Jingwei Zhou

Beijing University of Chinese Medicine Affiliated Dongzhimen Hospital

Weijing Liu

Beijing University of Chinese Medicine Affiliated Dongzhimen Hospital

Xueting Han

Beijing University of Chinese Medicine Affiliated Dongzhimen Hospital

Zhenjie Chen

Beijing University of Chinese Medicine Affiliated Dongzhimen Hospital

Qingqing Liu

Beijing University of Chinese Medicine Affiliated Dongzhimen Hospital

\section{Weihong Chen}

Beijing University of Chinese Medicine Affiliated Dongzhimen Hospital

\section{Mei Han}

Beijing University of Chinese Medicine

\section{Zhen Wang ( $\nabla$ zhenwangdzm@126.com)}

Beijing University of Chinese Medicine https://orcid.org/0000-0003-3433-6381

Study protocol

Keywords: Chinese herbal medicine, syndrome, Diabetic kidney disease, Randomized controlled trial 
Posted Date: June 28th, 2021

DOl: https://doi.org/10.21203/rs.3.rs-464664/v1

License: (c) (i) This work is licensed under a Creative Commons Attribution 4.0 International License. Read Full License 


\section{Abstract}

\section{Introduction}

Diabetic kidney disease (DKD) is a major microvascular complication of diabetes mellitus (DM) and the primary cause of end-stage renal disease. Routine medication might result in adverse effects which limit their usage. Laboratory studies have shown that Xiao Zheng San Jie (XZSJ) granules is effective in reducing the levels of urinary protein and blood creatinine in rats with DKD. Hence, we aim to conduct a clinical trial to determine the efficacy and safety of XZSJ granules among patients with DKD Stage IV, who present with the syndromes of qi deficiency and blood stasis. The purpose of this paper is to report the methodology of this trial.

\section{Methods and analysis}

This is a randomised, double-blind, placebo-controlled, multi-centre clinical trial. A total of 72 patients with DKD Stage IV who have the syndromes of qi deficiency and blood stasis will be randomly assigned to either intervention or placebo group. In addition to routine medication, the intervention group will be given XZSJ formula (in granules) and the control group will be given placebo for 12-weeks. Data collected at baseline as well as weeks 4,8 and 12 will be recorded in case report forms and subsequently entered into EpiData 3.1 electronic database. Adverse events will also be documented. Primary outcomes - estimated glomerular filtration rate, urinary albumin-to-creatinine ratio, and serum creatinine - will be assessed after 12 weeks.

\section{Ethics and dissemination}

This trial has been approved by the Research Ethics Committee of Dongzhimen Hospital (DZMEC-KY2018-60). Informed consent will be obtained from all participants. Results of this study will be disseminated to the public through academic conferences and peer-reviewed journals.

\section{Discussion}

This trial might provide information on an effective method for the treatment of DKD, especially for patients who present with the syndromes of qi deficiency and blood stasis. We hope that this trial will serve as a reference for the conception of high-quality Traditional Chinese Medicine trials in the future, which incorporate syndrome diagnosis into the study design.

\section{Trial registration}

Chinese Clinical Trial Registry, ChiCTR1900021391. Registered 18 February 2018, http://www.chictr.org.cn/showproj.aspx?proj=36106.

\section{Background}


Based on statistics obtained from the 9th edition of the International Diabetes Federation (IDF) Diabetes Atlas, there are currently 463 million adults with diabetes mellitus (DM) worldwide. If adequate measures are not taken, this number will increase to 578 million by 2030 and subsequently to 700 million by 2045[1]. Diabetic kidney disease (DKD) is the main microvascular complication of DM. Globally, it is the primary cause of chronic kidney disease (CKD), which could lead to end-stage renal disease (ESRD), and is also an important risk factor for cardiovascular disease in patients [2]. With the rapid economic development in China and lifestyle change in the Chinese population, the prevalence of DKD has been increasing rapidly following the rise in the number of patients with DM [3]. Currently, the number of CKD cases associated with DKD in China is estimated at 24.3 million, which has exceeded the number of CKD cases associated with glomerulonephritis [4], hence highlighting the need for effective management of DKD.

In addition to managing hypertension, hyperglycemia and dyslipidemia, inhibition of the reninangiotensin-aldosterone system (RAAS) has been the mainstay treatment for DKD. Many studies have shown that angiotensin-converting enzyme inhibitors (ACEls) or angiotensin receptor blockers (ARBs) can delay the progression of proteinuria and reduce the risk of cardiovascular events [5,6]. However, there is insufficient evidence about the efficacy of these drugs in patients without hypertension [7]. In addition, side effects associated with ACEls and ARBs (such as hyperkalemia, acute kidney injury and cough) $[8,9]$ limit the usage of these drugs. ACEls and ARBs are also contraindicated in pregnant women due to possible fetal renal damage [10]. Although studies have shown that new hypoglycemic drugs such as glucagon-like peptide-1 (GLP-1) receptor agonists, dipeptidyl peptidase-4 (DDP-4) inhibitors, and sodium glucose transporter 2 (SGLT2) inhibitors can delay the progression of proteinuria and ESRD [11-13], these clinical trials mainly recruited patients with a higher risk of cardiovascular events and did not use renal function as the primary endpoint. In addition, potential adverse events associated with these drugs, such as diabetic ketoacidosis, gastrointestinal discomfort and urinary tract infection [14-16] also limit their usage. Thus, there is an unmet need for the treatment of DKD.

Chinese herbal medicine (CHM) has a longstanding history in China and is an effective complementary therapy for improving the symptoms and quality of life of patients with DKD. According to Traditional Chinese Medicine (TCM) theory, patients at different stages of a disease can present with different syndromes ("Zheng"). In order to increase treatment efficacy, CHM should be tailored to fit the patients' syndrome. Results from TCM syndrome studies have shown that qi deficiency and blood stasis are predominant during DKD Stage IV. Xiao Zheng San Jie (XZSJ) formula (also known as Shenyan Fangshuai Formula) is a CHM formula curated by Professor Wang Yaoxian for patients with DKD, who present with the syndromes of qi deficiency and blood stasis syndrome. This formula is based on the "Zhengjia" theory by Professor Lv Renhe, a renowned TCM physician [17]. Preliminary studies involving rats with DKD have shown that XZSJ formula can reduce the levels of urinary protein and serum creatinine by inhibiting the TNF-a/NF-KBp65 signaling pathway [18]. XZSJ formula contains 6 herbs, namely astmgali radix, eucommiae cortex, sargassum, eupolyphaga, radix rhei et rhizome, and smilacis glabrae rhixoma, which can reduce inflammation, oxidation, improve hemodynamics, and protect vascular endothelial cells according to pharmacological studies [19-21]. 
There has been no randomised controlled trial evaluating the efficacy of XZSJ formula. Hence, we designed a randomised, double-blind, placebo-controlled, multi-centre clinical trial to evaluate the efficacy and safety of XZSJ formula in the treatment of DKD. This trial might serve as a reference for the conception of future TCM clinical trials.

\section{Trial objective}

This trial aims to assess the efficacy and safety of XZSJ formula for patients with DKD Stage IV who have qi deficiency and blood stasis syndrome.

\section{Methods/ Design}

\section{Study design and recruitment}

This is a randomised, double-blinded, placebo-controlled, multi-centre trial to evaluate the efficacy and safety of XZSJ granules in the treatment of patients with DKD Stage IV as well as qi deficiency and blood stasis syndrome. The protocol is guided by the Declaration of Helsinki, Good Clinical Practice (GCP) guidelines and the Standard Protocol Items: Recommendations for Interventional Trials (SPIRIT, 2013), see Additional file 1.

Before the implementation of the clinical trial, we obtained the written consent of the Ethics Committee. All subjects will be required to read carefully and sign the informed consent form before participating in this trial. The investigators should give the subjects sufficient time to understand the details of the clinical trial and answer as much as possible any questions related to the clinical trial in detail. Investigators will conduct the first screening for recruited subjects $7 \pm 3$ days before baseline. The content of the first follow-up visit mainly includes the general information of the subjects, reviewing the diagnosis, inclusion and exclusion criteria, the efficacy and safety observation. Subjects that fulfil the inclusion and exclusion criteria will be given standardised western conventional treatment for 7 days before the second screening. Eligible subjects will then be randomised in a 1:1 ratio to receive either XZSJ granules or placebo. After randomization, all participants will be evaluated at baseline as well as weeks 4 , 8 and 12.

Seventy-two subjects will be recruited from 6 hospitals in China, namely Shandong Provincial Hospital of Traditional Chinese Medicine, Shanxi Provincial Hospital of Traditional Chinese Medicine, Hebei Provincial Hospital of Traditional Chinese Medicine, The First Affiliated Hospital of Beijing University of Chinese Medicine Dongzhimen Hospital, The First Affiliated Hospital of Tianjin University of Chinese Medicine, and The First Affiliated Hospital of Henan University of Chinese Medicine (Table 1). Recruitment will be directed at patients with DKD and conducted by clinical staff at outpatient clinics or through recruitment brochures on hospital notice boards and WeChat application programmes.

\section{Diagnostic criteria}


We will recruit participants aged between 25 and 75 years, who meet the diagnostic criteria for DKD based on the National Kidney Foundation Kidney Disease Outcomes Quality Initiative (NKF-KDOQI) guidelines

[22], the Kidney Disease Improving Global Outcomes (KDIGO) 2012 Clinical Practice Guideline [23] and Chinese Diabetes Association Chinese Diabetic Kidney Disease Prevention and Treatment Clinical Guidelines [24]. For a diagnosis of DKD, patients with DM should have at least one of the following conditions: 1) Albuminuria (for microalbuminuria, out of three tests, two repeated tests performed within 3-6 months will need to produce abnormal results); 2) Diabetic retinopathy with CKD; 3) DKD confirmed by renal biopsy. If any of the following occurs, the chronic kidney disease is considered to be caused by other reasons and needs to be ruled out: 1 ) Shorter course (type 1 diabetes $<10$ years) or no diabetic retinopathy; 2) Estimated glomerular filtration rate decreased rapidly; 3 ) Urine protein increased rapidly or nephrotic syndrome appeared; 4) Refractory hypertension; 5) Active urine sediment (red blood cells, white blood cells or cell casts, etc.); 6) Complicated with symptoms or signs of other systemic diseases; 7) After treatment with ACEI or ARB, the eGFR decreased by more than $30 \%$ within 3 months; 8 ) Kidney ultrasound found abnormal.

After diagnosis of DKD, we will refer to the stage differentiation of diabetic nephropathy proposed by Danish scholar Mogensen, Combined with the stage differentiation of CKD by recommendations of the KDIGO guideline: the CKD staging (G1 stage: eGFR $\geq 90 \mathrm{~mL} / \mathrm{min} / 1.73 \mathrm{~m} 2, \mathrm{G} 2$ stage: $90>\mathrm{eGFR} \geq 60 \mathrm{~mL} / \mathrm{min}$ / 1.73m2, G3 stage: $60>\mathrm{eGFR} \geq 30 \mathrm{~mL} / \mathrm{min} / 1.73 \mathrm{~m} 2$, G4 stage: $30>\mathrm{eGFR} \geq 15 \mathrm{~mL} / \mathrm{min} / 1.73 \mathrm{~m} 2$, G5 stage: eGFR $<15 \mathrm{~mL} / \mathrm{min} / 1.73 \mathrm{~m} 2$ ) ; The albuminuria staging (A1 stage: Urinary albumin-to-creatinine ratio (UACR) $<30 \mathrm{mg} / \mathrm{g}$, A2 stage: UACR $30 \otimes 300 \mathrm{mg} / \mathrm{g}$, A3 stage: UACR $>300 \mathrm{mg} / \mathrm{g}$ ), to determine the staging criteria of DKD: 1) DKD stage I: High filtration rate stage, UACR $<30 \mathrm{mg} / \mathrm{g}$, eGFR is much greater than $90 \mathrm{~mL} / \mathrm{min} / 1.73 \mathrm{~m} 2$; 2) DKD stage II: Normal proteinuria stage, UACR $<30 \mathrm{mg} / \mathrm{g}, \mathrm{eGFR} \geq 90 \mathrm{~mL} / \mathrm{min}$ / $1.73 \mathrm{~m} 2$; 3) DKD stage $₫$ : Microalbuminuria stage, UACR: $30 \rrbracket 300 \mathrm{mg} / \mathrm{g}$, eGFR $\geq 90 \mathrm{~mL} / \mathrm{min} / 1.73 \mathrm{~m} 2$; 4)

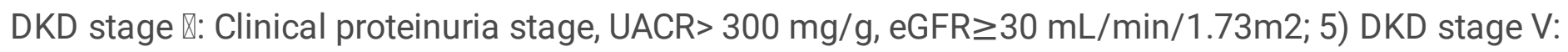
End-stage renal failure, eGFR $<30 \mathrm{~mL} / \mathrm{min} / 1.73 \mathrm{~m} 2$. Patients with DKD consistent with Mogensen Stage IV (UACR $>300 \mathrm{mg} / \mathrm{g}, e G F R \geq 30 \mathrm{~mL} / \mathrm{min} / 1.73 \mathrm{~m} 2$ ), will be considered in this trial.

Patients should also meet the diagnostic criteria for qi deficiency and blood stasis syndromes, which were adapted from the TCM Clinical Diagnosis and Treatment Plan for 95 Diseases in 22 Specialties, issued by the State Administration of Traditional Chinese Medicine of China. Patients should present with at least 3 of the following signs and symptoms for a diagnosis of qi deficiency to be established: 1) fatigue, 2) exhaustion after talking, 3) prone to sweating, 4) swollen tongue with teeth marks, 5) weak pulse. Patients should present with at least 2 of the following signs and symptoms for a diagnosis of blood stasis syndrome to be established: 1) sharp pain in a fixed location which worsens at night, 2) numbness or hemiplegia, 3) dry, scaly skin, 4) petechiae, purplish lips and tongue, dark spots on the tongue, or prominent sublingual vessel. TCM syndrome diagnosis will be conducted independently by 2 TCM physicians with experience in nephrology. The patient will be recruited if both physicians have similar diagnosis. A third physician will be consulted should there be differing diagnoses. 


\section{Inclusion/Exclusion criteria}

\section{Inclusion criteria}

Patients should have DKD consistent with Mogensen Stage IV, age between 25 and 75 years, eGFR of 30$89 \mathrm{~mL} / \mathrm{min} / 1.73 \mathrm{~m} 2$ without dialysis, 24 -hour urinary protein $(24 \mathrm{~h}-\mathrm{UP})<3 \mathrm{~g}$, blood pressure $\leq$ $160 / 90 \mathrm{mmHg}$ and hemoglobin A1c (HbA1c) levels $\leq 9 \%$.

\section{Exclusion criteria}

We will exclude patients who have had severe infection, anemia, electrolyte imbalance, or acute complications of DM in the past 4 weeks; severe cardiac, cerebral, hepatic, or hemorrhagic diseases (e.g. cerebral infarction, cerebral hemorrhage, transient ischemic attack, myocardial infarction, unstable angina, heart failure, hepatic inadequacy with aspartate transaminase (AST) or alanine aminotransferase (ALT) levels more than twice the normal upper limit) in the past 3 months; consumed corticosteroids or immunosuppressants in the past 3 months; or had renal transplantation. We will also exclude patients with oliguria, anuria, severe edema, massive pleural or peritoneal effusion, active hepatitis $B$, mental disorders, female patients who are pregnant or breastfeeding, as well as patients with an allergy to the trial drug or who are currently participating in other clinical studies.

\section{Handling of withdrawal and dropout}

Participants will be allowed to withdraw from the trial at any time. Reason and date of withdrawal will be recorded in the CRF. Investigators will withdraw participants from the trial, should participants have an occurrence of diseases or conditions mentioned in the exclusion criteria, severe complications and/or general health deterioration, serious adverse events, rapidly increasing levels of serum creatinine (more than $50 \%$ of baseline) or ESRD, violate the trial protocol, or loss to follow-up to ensure the safety and quality of the trial.

\section{Randomisation and blinding}

Eligible participants will be allocated to either intervention or control group in a 1:1 ratio. Participants will be referred to an administrator, who is not involved in recruitment and follow-up of participants. The administrator will have the randomisation sequence numbers, which will be pre-generated by Beijing University of Chinese Medicine Centre for Evidence-based medicine using Statistical Analysis System (SAS version 9.2). Sequence numbers will be kept in sealed, opaque envelopes, which will be opened sequentially and revealed to participants on the day of randomisation. Participants, investigators and statisticians will be blinded to allocation to ensure allocation concealment.

\section{Intervention}

Eligible patients will be administered with routine treatment for DKD as per recommendations by KDIGO 2012 Clinical Practice Guideline [23] and Chinese Diabetes Association 2019 Chinese Diabetic Kidney 
Disease Prevention and Treatment Clinical Guidelines [24]. These include regulation of blood glucose to maintain HbA1c levels $£ 9 \%$ and regulation of blood pressure at $£ 140 / 90 \mathrm{mmHg}$, except for individuals aged 60 years or older, for whom the threshold for systolic blood pressure will be $£ 160 \mathrm{mmHg}$. Patients assigned to the intervention and control arm will receive XZSJ granules and placebo respectively. Patients will be required to consume one pack twice daily for 12 weeks, and will be advised against using other modes of TCM treatment during this period. XZSJ granules consists of astmgali radix, eucommiae cortex, sargassum, eupolyphaga, radix rhei et rhizome, and smilacis glabrae rhixoma (Table 2), while placebo granules are made from dextrin. Both types of granules will be provided by Beijing Tcmages Pharmaceutical Co. Ltd. (Beijing, China) and will have similar packaging, colour and taste to prevent potential unblinding. Designated drug distributors will distribute either XZSJ granules or placebo to participants at each visit and record the serial number, number of bags taken as well as number of bags returned for accountability.

\section{Data collection and follow-up plan}

The standardized CRF designed for this study will be used to collect data at each scheduled visit, which will be kept in a locked cabinet. The subject information is illegal or unauthorized given to access, disclosure and dissemination. Clinical trial data will be reasonably recorded, processed, and maintained to ensure confidentiality of records and subject information. Subjects will be evaluated during the enrolment (-1week), baseline (0week), and double-blind treatment period (4, 8 and 12 weeks). Each followup visit should take place within \pm 3 days of the scheduled time. The screening visit will collect background information during the screening period, including demographic indicators and general clinical data. Demographic indicators include date of birth, nationality, sex, height and weight. General clinical data include previous medical history, treatment history, and information related to the diagnosis of the disease (including UACR, Scr, eGFR, etc.), vital signs and physical examination, concomitant disease, and combined medication. From the second follow-up visit to the end of the trial, each follow-up collecting information will include the relevant data of TCM syndromes, vital signs, physical examination and relevant laboratory examination results in the trial. Hemoglobin A1c (HbA1c) and quality of life scales will be measured and collected during the screening period and 12 weeks. The follow-up visit schedule of data collection is shown in Figure1 and Figure 2. In this trial, we will adopt the form of paper/electronic data double entry. All CRF data will be imported into the electronic data management system EpiData 3.1, which is password protected to ensure confidentiality of information. Two investigators will independently enter the data and any discrepancies will be resolved to ensure accuracy. Problems found during the data entry process will be reported to the monitor, who would raise a query to the investigator. All queries will be documented and kept for future reference.

\section{Primary and secondary outcomes}

Primary outcome measures of the clinical trial include UACR, Scr and eGFR. These results will be measured at baseline as well as weeks 4,8 and 12 after randomization. Secondary outcome measures include hemoglobin ( $\mathrm{Hb}$ ), albumin (Alb), Kidney Disease Quality of Life-Short Form (KDQOL-SF) [25], 
EuroQol Five Dimensions questionnaire (EQ-5D) [26] and the TCM syndrome score, which will be recorded at baseline as well as weeks 4, 8 and 12. EQ-5D consists of the EQ-5D health description system which assesses mobility, self-care, usual activities, pain/discomfort and anxiety/depression, as well as the EQVAS, which assesses quality of health, with higher scores indicating better health. KDQOL-SF scale consists of the short form health survey (SF-36) and kidney disease-targeted scales (KDTA). SF-36 assesses for physiological function, physical pain, general health, mental health, emotional function, social function, energy and health changes. KDTA assesses for symptoms, effects and burden of kidney disease, work status, cognitive function, social quality, sexual function, sleep and social support. Higher scores on KDQOL-SF and EQ-5D indicate better health status. TCM syndromes are graded based on the severity of symptoms related to qi deficiency and blood stasis, with higher scores reflecting more severe symptoms. Changes in syndrome scores will be assessed to be significant (reduction in score by ${ }^{370 \%}$ ), effective (reduction in score by ${ }^{3} 30 \%$ ) or invalid (reduction in score by $<30 \%$ ).

All laboratory indicators including UACR, Scr, $\mathrm{Hb}$ and Alb will be tested by Dongzhimen Hospital. eGFR will be calculated using the CKD-EPI formula. Differences in primary and secondary outcomes will be calculated and used for analysis to reduce biasedness caused by differences in baseline data. (change rate $=$ change value $/$ baseline detection, change value $=$ baseline detection value-detection value at the Nth week of medication).

\section{Safety}

For this study, an adverse event (AE) is any unfavorable sign (including abnormal laboratory finding), symptom or disease which might or might not have a causal relationship with the intervention. Events related to physiological changes (e.g. menopause, menstrual cramps) will not be considered as an AE. Serious adverse event (SAE) is any untoward medical occurrence that leads to death, is life-threatening, a congenital defect, causes permanent or severe disability/incapacity or requires hospitalisation or prolongation of hospitalisation. In the event of an $\mathrm{AE}$, the time of onset, duration, severity, intervention measures and outcome will be recorded in the CRF. Patients will be followed-up for their AE till resolution. In the event of a SAE, the SAE form will be documented and submitted to the research unit, ethics committee and project unit of Dongzhimen Hospital within 24 hours. Appropriate treatment for any SAEs will be administered for participants and the trial will be suspended till further notice.

Adverse drug reaction (ADR) refers to any harmful or undesired effects caused by the trial drug at any dose, such as exacerbation of existing illnesses and injuries or untoward events caused by overdose, abuse, withdrawal, allergy and toxicity. If there is even one reasonable possibility of a causal relationship between trial drugs and ADRs, the association cannot be ruled out. All ADRs will be recorded even if there is only one reasonable possibility of a causal relationship. Patients will be followed-up for their ADRs till resolution.

\section{Sample size justification}


As this is the first study to explore the efficacy of XZSJ granules on patients with DKD, we plan to recruit 60 subjects for both arms for a preliminary analysis. Allowing for a dropout rate of $20 \%$, a total of 72 subjects will be needed, with 12 subjects being recruited from each centre. We hope that results of this trial will provide data support for the calculation of sample sizes for future large-scale clinical trials involving patients with DKD.

\section{Quality control}

Prior to trial commencement, all investigators will receive training in Good Clinical Practice (GCP) and Standard Operation Procedures (SOPs) constructed for this trial to ensure quality of study procedures. Principal investigators as well as the supervisory and administrative departments of the pharmaceutical company will oversee implementation of the trial. All centres will be actively involved in quality control during data processing. The study team will conduct interim monitoring of trial implementation, data quality and integrity of the data to ensure that all processes comply with GCP and SOPs.

\section{Statistical analysis}

Statistical analysis will be conducted using Social Science Statistical Software Package (SPSS) Version 22.0 by a statistician who is blinded to treatment allocation. Continuous variables will be expressed as mean \pm standard deviation (SD), while categorical variables will be expressed as $n(\%)$. $t$-test or $t^{\prime}$-test will be used for the analysis of continuous variables with a normal distribution, based on results of homogeneity of variance. Mann-Whitney-Wilcoxon test will be used for the analysis of continuous variables without a normally distribution. Statistical analysis will assume a two-sided test and $95 \%$ confidence interval. $\mathrm{P}<0.05$ will be considered statistically significant.

\section{Ethics and dissemination}

This trial has been registered at Chinese Clinical Trial Registry http://www.chictr.org.cn/. The study will be conducted in compliance with the Declaration of Helsinki and GCP guidelines. Data of this trial will be uploaded into ResMan at http://www.medresman.org/ and posted on Chinese Clinical Trial Registry. Results of this trial will be disseminated to the public through academic conferences and peer-reviewed journals.

\section{Discussion}

Traditional Chinese medicine has a curative effect and is able to improve the symptoms and the quality of life of patients with DKD. As syndrome differentiation is an important guiding concept in TCM, where a disease can present with different syndromes at different stages [28], it is important to tailor TCM treatment methods to suit patients' syndromes for better treatment effects. Previous clinical trials did not consider TCM syndromes of patients at different stages of the disease. This trial incorporates the diagnosis of qi deficiency and blood stasis syndromes into its inclusion criteria, to align with the intended treatment effect of XZSJ granules - benefiting qi for activating blood circulation, and softening and 
resolving hard mass. Results from this trial might highlight and provide evidence of the importance of TCM syndrome diagnosis in clinical practice and clinical studies.

Although this clinical trial attempts to rectify the weaknesses of previous trials by incorporating TCM syndrome diagnosis, some limitations are foreseen. Firstly, the sample size might not be large enough. However, as this is the first randomised controlled trial evaluating the efficacy of XZSJ granules in the treatment of DKD, we hope that results from this trial will serve as a reference for the calculation of appropriate sample sizes of future clinical trials. Secondly, although we attempted to reduce discrepancies in TCM syndrome diagnosis by using standardized criteria, patients might present with accompanying syndromes that might add to the complexity of syndrome diagnosis. More relevant studies are needed to elucidate the TCM syndromes in patients with DKD.

Taken together, this clinical trial might provide information on an effective method for the treatment of DKD, especially for patients who present with the syndromes of qi deficiency and blood stasis.

\section{Trial status}

Recruitment for the trial was planned to start on January 2019 and last until January 2020. Due to the COVID-19, the trial is delayed and we are currently recruiting participants. The current protocol is version 2.0 and is dated 4 January 2019.

\section{Declarations}

\section{Ethics approval and consent to participate}

This trial has been registered at Chinese Clinical Trial Registry (http://www.chictr.org.cn/, ChiCTR1900021391) and approved by the Ethics Committee of Dongzhimen Hospital Affiliated to Beijing University of Chinese Medicine (approval number DZMEC-KY-2018-60) as well as by the local Ethics Committee of each center, see Additional file 2. Informed consent will be signed and obtained from all participants.

\section{Consent for publication}

Not applicable.

\section{Availability of data and material}

The data used or analysed are from previously reported studies and datasets, which have been cited.

\section{Competing interests}

All authors declare that they have no conflicts of interest and there is no financial and other competing interests for principal investigators for the overall trial and each study site. 


\section{Funding Statement}

This study is supported by the National Key Project for Drug Discovery (No. 2017ZX09304019). the Natural Science Foundation of China (Grant No. 81804032) and the Natural Science Foundation of China (Grant No. 81774298), see Additional file 3. The funder has not participated in the design of the study and collection, analysis, and interpretation of data and in writing the manuscript.

\section{Authors' contributions}

SNA and YZH contributed equally to this work as co-first authors, SNA, YZH and GYB wrote the first draft of protocol and write the manuscript. ZJW and YXW was the principal investigator and leader of the project. WJL involved in designing and helped to work out the design of the study protocol. XTH, ZJC, QQL, WHC revised the protocal and provided professional guidance. MH provided advize on statistical methods of the study design. ZW is responsible for the implementation of research and provided critical revisions to the protocol. All authors read and approved the final version of the manuscript.

\section{References}

1. International Diabetes Federation. IDF Diabetes Atlas. 9th ed. Brussels: International Diabetes Federation; 2019.

2. Afkarian M, Zelnick LR, Hall YN, et al. Clinical Manifestations of Kidney Disease Among US Adults with Diabetes, 1988-2014. JAMA. 2016;316(6):602-10.

3. Chan JC, Zhang Y, Ning G. Diabetes in China: a societal solution for a personal challenge. Lancet Diabetes Endocrinol. 2014;2(12):969-79.

4. Zhang L, Long J, Jiang W, et al. Trends in Chronic Kidney Disease in China. N Engl J Med. 2016;375(9):905-6.

5. Lewis EJ, Hunsicker LG, Clarke WR, Berl T, Pohl MA, Lewis. JB, et al.

6. Renoprotective effect of the angiotensin-receptor antagonist irbesartan.

7. in patients with nephropathy due to type 2 diabetes. $\mathrm{N}$ Engl J Med.

8. 2001.;345(12):851-60.

9. Brenner BM, Cooper ME, de Zeeuw D, et al. Effects of losartan on renal and cardiovascular outcomes in patients with type 2 diabetes and nephropathy. N Engl J Med. 2001;345(12):861-9.

10. Haller $\mathrm{H}$, Ito $\mathrm{S}$, Izzo JL Jr, et al. Olmesartan for the delay or prevention of microalbuminuria in type 2 diabetes. N Engl J Med. 2011;364(10):907-17.

11. Investigators ONTARGET, Yusuf S, Teo KK, et al Telmisartan, ramipril, or both in patients at high risk for vascular events. N Engl J Med. 2008;358(15):1547-1559. [93] Fried Fried LF, LF, [9] Emanuele N, Zhang. $\mathrm{JH}$, et al. Combined angiotensin inhibition for the treatment of diabetic nephropathy. $\mathrm{N}$ Engl $\mathrm{J}$ Med. 2013;369(20):1892-1903. 
12. Pucci M, Sarween N, Knox E, et al. Angiotensin-converting enzyme inhibitors and angiotensin receptor blockers in women of childbearing age: risks versus benefits[J]. Expert Rev Clin Pharmacol,2015,8(2):221-231.

13. Mosenzon O, Leibowitz G, Bhatt DL, et al. Effect of Saxagliptin on Renal Outcomes in the SAVORTIMI 53 Trial. Diabetes Care. 2017;40(1):69-76.

14. Marso SP, Daniels GH, Brown-Frandsen K, et al. Liraglutide and Cardiovascular Outcomes in Type 2 Diabetes. N Engl J Med. 2016;375(4):311-22.

15. Neal B, Perkovic V, Mahaffey KW, et al. Canagliflozin and Cardiovascular and Renal Events in Type 2 Diabetes. N Engl J Med. 2017;377(7):644-57.

16. Fralick M, Schneeweiss S, Patorno E. Risk of Diabetic Ketoacidosis after Initiation of an SGLT2 Inhibitor. N Engl J Med. 2017;376(23):2300-2.

17. Fadini GP, Bonora BM, Mayur S, Rigato M, Avogaro A. Dipeptidyl peptidase-4 inhibitors moderate the risk of genitourinary tract infections associated with sodium-glucose co-transporter-2 inhibitors. Diabetes Obes Metab. 2018;20(3):740-4.

18. Husain M, Birkenfeld AL, Donsmark M, et al. Oral Semaglutide and Cardiovascular Outcomes in Patients with Type 2 Diabetes. N Engl J Med. 2019;381(9):841-51.

19. Liu Shangjian W, Hui W, Yaoxian L, Yuanyuan Lu, Renhe. A preliminary study on the theory of "kidney collateral miniature syndrome". Chinese Journal of Basic Medicine in Traditional Chinese Medicine,2009,15(09): 649-650.

20. Lv J, Wang Z, Wang Y, et al. Renoprotective Effect of the Shen-Yan-Fang-Shuai Formula by Inhibiting TNF-a/NF-KB Signaling Pathway in Diabetic Rats. J Diabetes Res. 2017; 2017: 4319057.

21. Li X, Qu L, Dong Y, et al. A review of recent research progress on the astragalus genus. Molecules. 2014;19(11):18850-80.

22. Liu H, Yan Y, Zhang F, Wu Q. The Immuno-Enhancement Effects of Tubiechong (Eupolyphaga sinensis) Lyophilized Powder in Cyclophosphamide-Induced Immunosuppressed Mice. Immunol Invest. 2019;48(8):844-59.

23. Gao CH, Qu JQ, Zhou XY, Gao TS. Iodine-Rich Herbs and Potassium lodate Have Different Effects on the Oxidative Stress and Differentiation of TH17 Cells in lodine-Deficient NOD.H-2h4 Mice. Biol Trace Elem Res. 2018;183(1):114-22.

24. National Kidney Foundation. KDOQI Clinical Practice Guideline for Diabetes and CKD. 2012 Update [published correction appears in Am J Kidney Dis. 2013 Jun;61(6):1049]. Am J Kidney Dis. 2012;60(5):850-886.

25. Kidney Disease: Improving Global Outcomes (KDIGO) CKD Work Group. KDIGO 2012 clinical practice guideline for the evaluation and management of chronic kidney disease. Kidney Int Suppl. 2013;31(1):1-150.

26. Microvascular Complications Group of Diabetes Branch of Chinese Medical Association. Clinical guidelines for the prevention and treatment of diabetic kidney disease in China. Chinese Journal of Diabetes. 2019;11(01):15-28. 
27. Abd ElHafeez S, Sallam SA, Gad ZM, et al. Cultural adaptation and validation of the "Kidney Disease and Quality of Life-Short Form (KDQOL-SF ${ }^{\mathrm{TM}}$ ) version 1.3" questionnaire in Egypt. BMC Nephrol. 2012;13:170.

28. Wang H, Kindig DA, Mullahy J. Variation in Chinese population health related quality of life: results from a EuroQol study in Beijing, China. Qual Life Res. 2005;14(1):119-32.

29. Guo J, Chen H, Song J, et al. Syndrome Differentiation of Diabetes by the Traditional Chinese Medicine according to Evidence-Based Medicine and Expert Consensus Opinion. Evid Based Complement Alternat Med. 2014; 2014: 492193.

30. Sun Weiwei L, Shangjian L, Zhongjie, et al. Professor Wang Yaoxian's Trinity on the Treatment of Chronic Kidney Disease. Chinese Journal of Traditional Chinese Medicine, 2014, 29(09): 2820-2822

\section{Tables}

Table 1 Hospitals participating in this clinical trial

\section{Code Hospitals}

01 The First Affiliated Hospital of Beijing University of Chinese Medicine, Dongzhimen Hospital

02 The First Affiliated Hospital of Tianjin University of Chinese Medicine

03 The First Affiliated Hospital of Henan University of Chinese Medicine

04 Shandong Provincial Hospital of Traditional Chinese Medicine

05 Hebei Provincial Hospital of Traditional Chinese Medicine

06 Shanxi Provincial Hospital of Traditional Chinese Medicine

Table 2 Composition and proportion of XZSJ granules

\begin{tabular}{llll} 
Chinese name & English name & Origin & Weight (\%)* \\
\hline Huangqi & Astmgali radix & The dried root or rhizome & 22.2 \\
& & & \\
\hline Duzhong & Eucommiae cortex & The dried tree bark & 22.2 \\
\hline Haizao & Sargassum & The dried body & 22.2 \\
\hline Tubiechong & Eupolyphaga & The dried body & 7.4 \\
\hline Dahuang & Radix rhei et rhizome & The dried root & 3.7 \\
\hline Tufuling & Smilacis glabrae rhixoma & The dried root & 22.2
\end{tabular}

\section{Figures}




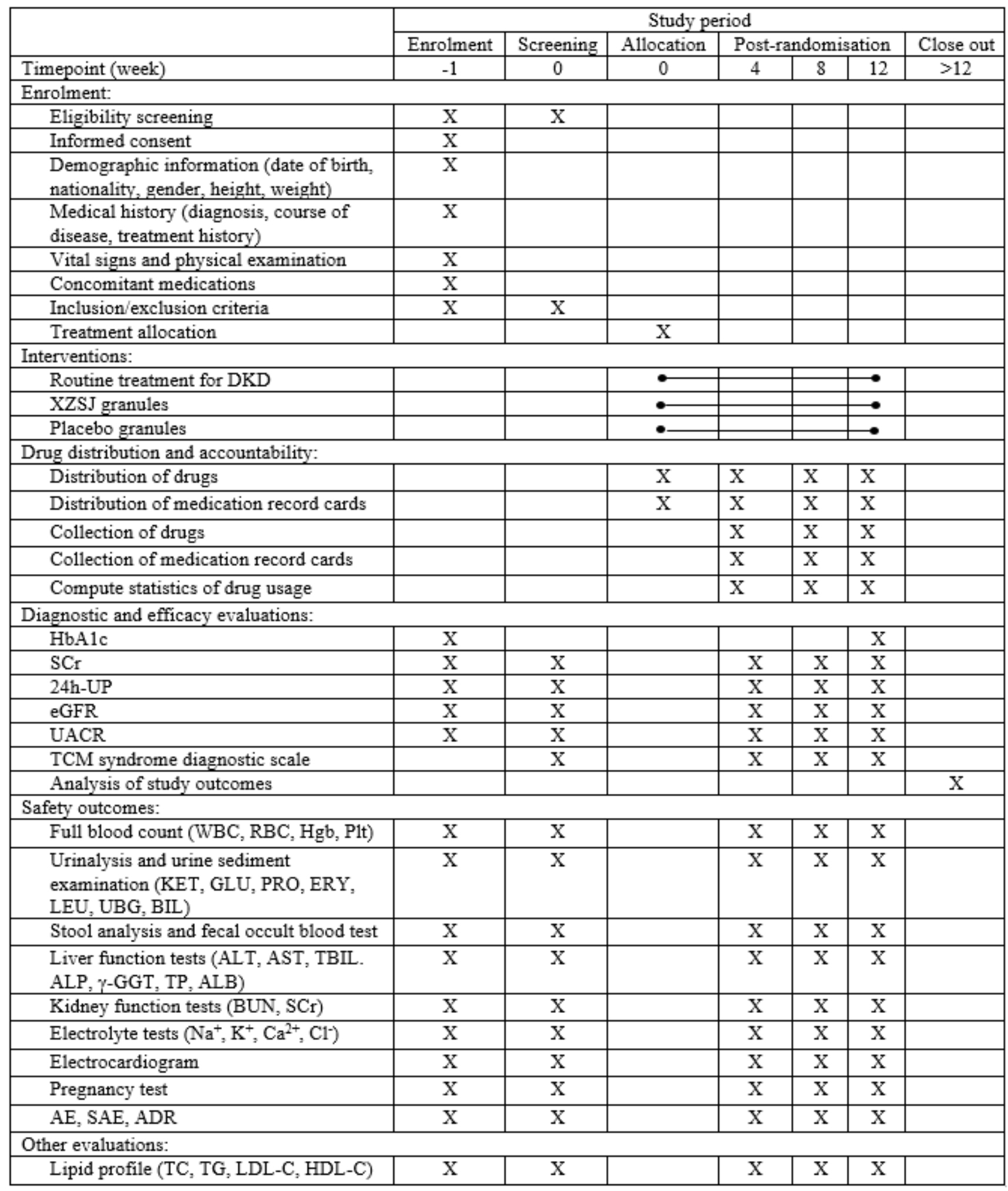

\section{Figure 1}

SPIRIT figure for the schedule of enrolment, screening, interventions and assessments. Abbreviations: ADR: adverse drug reaction, AE: adverse event, ALB: albumin, ALP: alkaline phosphatase, ALT: alanine aminotransferase, AST: aspartate transaminase, BIL: bilirubin, BUN: blood urea nitrogen, DKD: Diabetic kidney disease, eGFR: estimated glomerular filtration rate, ERY: erythrocytes, GLU: glucose, HbA1c: Hemoglobin A1C, HDL-C: high density lipoprotein cholesterol, Hgb: hemoglobin, KET: ketones, LDL-C: low 
density lipoprotein, cholesterol, LEU: leukocytes, PIt: platelets, PRO: protein, RBC: red blood cells, SAE: serious adverse event, SCr: serum creatinine, TBIL: total bilirubin, TC: total cholesterol, TCM: Traditional Chinese Medicine, TGL triglycerides, TP: total protein, UACR: urine albumin-to-creatinine ratio, UBG: urobilinogen, WBC: white blood cells, XZSJ: Xiao Zheng San Jie, 24h-UP: 24-hour urine protein, y-GGT: gamma-glutamyltransferase

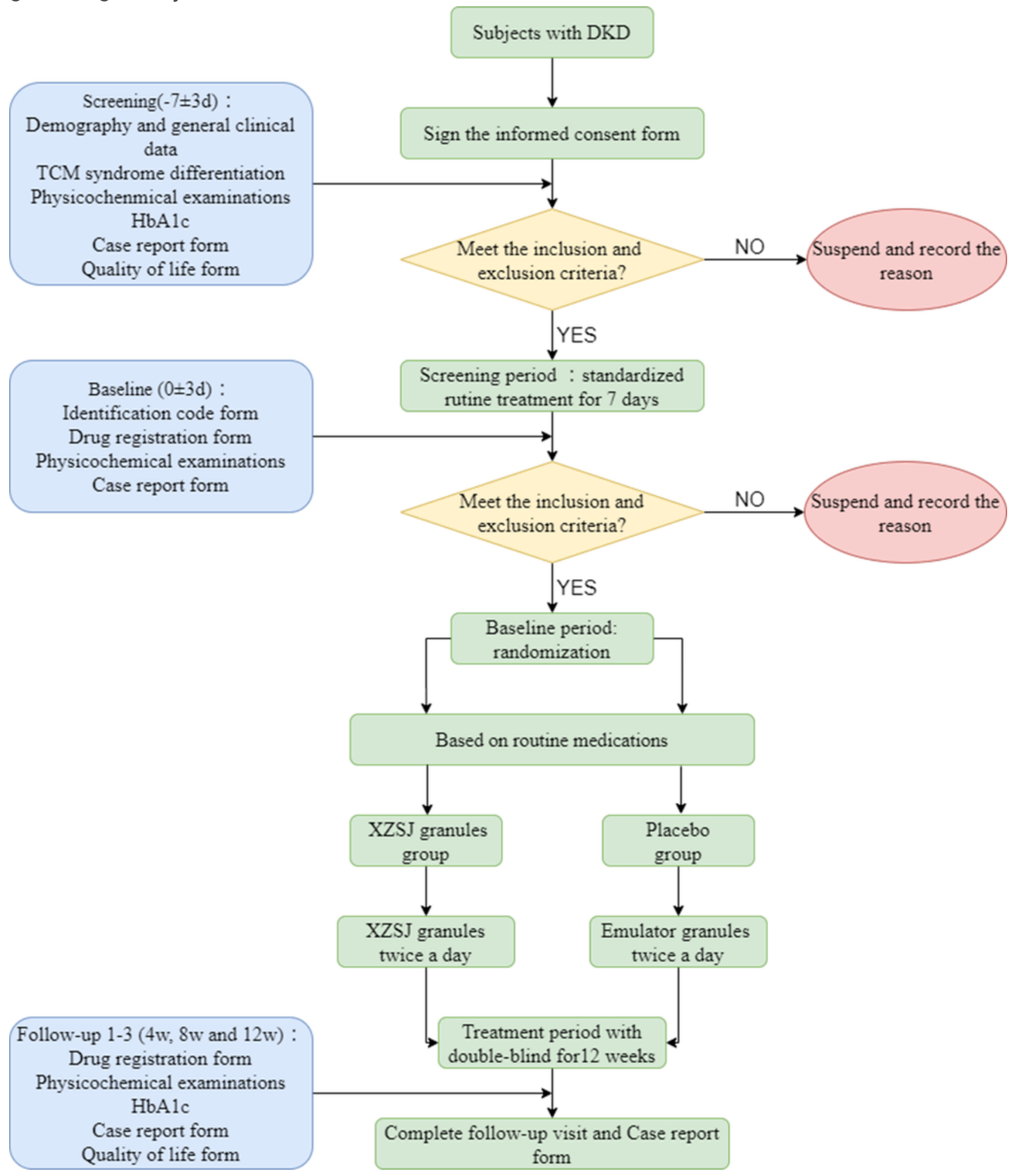

Figure 2 
Clinical trial workflow

\section{Supplementary Files}

This is a list of supplementary files associated with this preprint. Click to download.

- Additionalfile1SPIRIT2013.pdf 